\section{A Spatial Beam Splitter Consisting of a Near-Zero Refractive Index Medium}

Ruey-Bing Hwang, Neng-Chieh Hsu, and Cheng-Yuan Chin

\begin{abstract}
A novel metamaterial-based beam splitter is demonstrated in this communication. The metamaterial is a three-dimensional $(3 \mathrm{D})$ fishnet structure arranged in a two-dimensional (2 D) square lattice. According to Snell's law, the refracted wave from medium into air tends to be normal to the interface if the effective refractive index of medium is approximately zero. In light of this concept, the study presents a metamaterial with nearzero effective refractive index and an internal line source inside as excitation. Metallic flares with specific tilting angle were installed at four sides of the beam splitter to reduce reflection coefficient and match the impedance of the fishnet structure and free space. In addition to calculating the phase relation of eigenwaves in the metamaterial, this study measures the radiation patterns to verify its spatially beam-splitting characteristics.
\end{abstract}

Index Terms-Metamaterial, near-zero refractive index medium, periodic structures, spatial beam splitter.

\section{INTRODUCTION}

Metamaterial is an artificially engineered material that obtains its unusual electromagnetic properties from structure rather than its composition. Metamaterial is generally constructed by embedding specific inclusions such as periodic structures in a host medium. Previous studies have applied metamaterial in waveguides and antenna designs [1], [2]. A metamaterial with a near-zero effective refractive index can reshape the far-field pattern of an embedded antenna. A matched zero-index slab can also transform curved wave fronts into planar ones [3]. Metamaterial made of wire medium has been studied intensively and particularly on its effective refractive index, permittivity, and permeability. A structure composed of metallic mesh wires, which has very small electrical length in the period and wire thickness, can be treated as a homogeneous medium with a low plasma frequency [4]. Regarding the optical domain, a dielectric medium embedded with metallic nanoparticles and nano-wires has zero effective permittivity, creating band gaps [5]. Moreover, split ring resonators can produce an effective negative permeability over a microwave frequency band [6]. The first lefthanded metamaterial in microwave frequency was developed while the extraordinary refraction phenomenon was also demonstrated [7]. Metamaterials with both negative permittivity and permeability over an overlapping near-infrared wavelength range have a low loss negative-refractive-index [8], [9]. A three-dimensional optical metamaterial made of cascaded "fishnet" structures has a negative index within wide-band range [10]. Some researchers also used the effective medium method to consider the metamaterial slab as a uniform medium. Single-mode approximation can mathematically extract the effective parameters using the reflection- and transmission-coefficients of the metamaterial slab [11]. Antenna design benefits from the specific properties of metamaterial. For instance, a metamaterial consisting of six identical metallic

Manuscript received September 06, 2010; revised March 17, 2011; accepted July 02, 2011. Date of publication September 19, 2011; date of current version January 05,2012 . This work was supported by the National Science Council under Contract NSC 100-2221-E-009-138.

The authors are with the Department of Electrical Engineering, National Chiao-Tung University, 300 Hsinchu, Taiwan (e-mail: raybeam@mail.nctu. edu.tw).

Color versions of one or more of the figures in this communication are available online at http://ieeexplore.ieee.org.

Digital Object Identifier 10.1109/TAP.2011.2167913

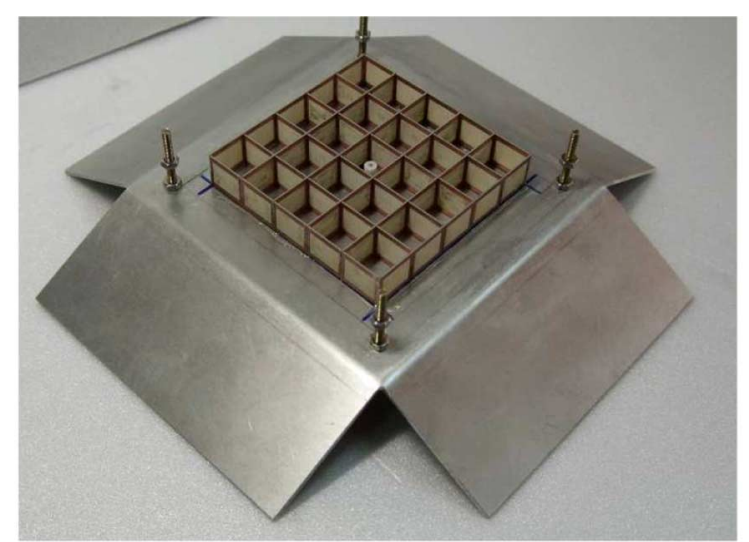

(a)

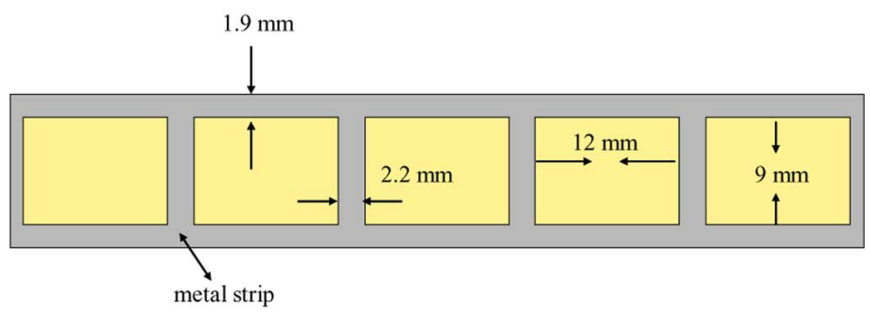

(b)

Fig. 1. Structure configuration: (a) photo of the $3 \mathrm{D}$ fishnet metamaterial, and (b) front view of the fishnet structure.

grids with a square lattice embedded in a foam [12]. They placed a monopole source in the middle of the structure, and a metal plate on the bottom of structure to control the emission. Experimental and numerical analysis proves that this metamaterial can modify the emission of an embedded source. Researchers have proposed and analyzed an epsilon-near-zero metamaterial for tailoring the phase of radiation pattern of arbitrary sources for some canonical geometries [13]. Other researchers studied a strongly modulated photonic crystal with an effective refractive index controllable by the band structure [14]. Experimental results prove that such a metamaterial can modify the emission of an embedded source and enhance its gain and directivity [15].

\section{Structure CONFIGURATION}

Fig. 1(a) shows the two metallic parallel plates sandwiching the fishnet structure. The numbers of the unit cell along the $x$ - and $y$-directions are both 5; the periods (or lattice constant) along the $x$ - and $y$-directions are $d_{x}(14.2 \mathrm{~mm})$ and $d_{y}(14.2 \mathrm{~mm})$, respectively. Each row or column of the $3 \mathrm{D}$ fishnet structure was constructed by the building block depicted in Fig. 1(b). Photo lithography and chemical etching processes were used to print a jungle-gym-like pattern on the dielectric substrate RO4003 with a relative dielectric constant of 3.55 and a thickness of $0.508 \mathrm{~mm}$. The same pattern was printed on both sides of the dielectric substrate. This figure also shows the dimensions of the metal strip. The metallic parallel plates were made of aluminum measuring $1.6 \mathrm{~mm}$ thick, and the distance between them was $h(12.8 \mathrm{~mm})$. The experiments in this study excited cylindrical wave with a coaxial probe in this structure to observe its beam-splitting characteristic. 


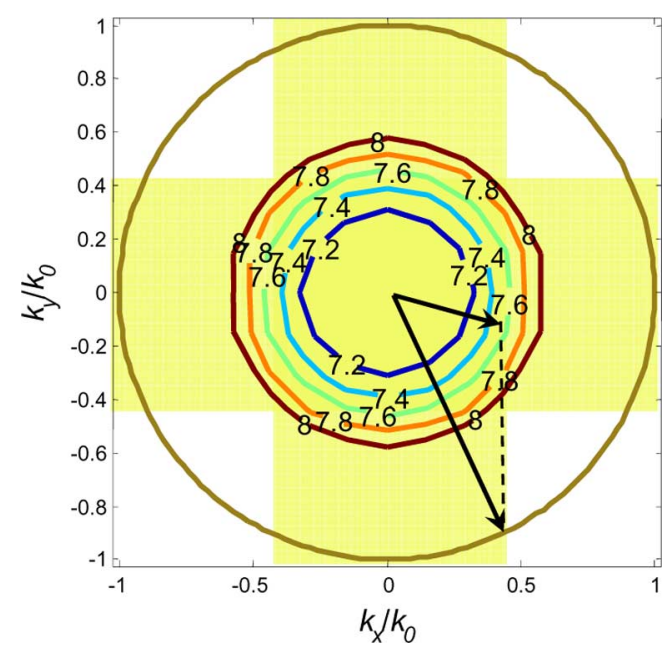

Fig. 2. Phase relation of waves propagating in the metamaterial.

\section{Characterization OF THE Metamaterial}

\section{A. Phase Relation}

When analyzing a periodic structure, the dispersion (or phase) relation of the eigen-wave supported in the medium is an important issue. The boundary conditions of the top and bottom surfaces are perfect electric conductors. Since the metamaterial is assumed to have infinite $2 \mathrm{D}$ periodicity, the phase differences along the $x$ - and $y$-directions can be expressed as $k_{x} d_{x}$ and $k_{y} d_{y}$ respectively, where phase constants $k_{x}$ and $k_{y}$ must comply with the Bloch-Floquet theorem, namely, the periodic boundary condition. Therefore, it is possible to solve the eigen-value problem of the closed structure using the finite-element method. Notice that the eigen-value (frequency) obtained corresponds to a propagating mode subject to every given combination of phase constants $k_{x}$ and $k_{y}$. After iterating the phase constants, it is possible to determine the phase relation (the relationship among frequency, $k_{x}$ and $k_{y}$ ). Fig. 2 shows a contour map of the phase relation, in which the horizontal and vertical axes represent the normalized phase constants $\left(k_{x} / k_{o}\right.$ and $\left.k_{y} / k_{o}\right)$ along the $x$ - and $y$-axis, respectively. This figure also reflects the symmetric properties of the unit cell. The colored circles with smaller radii represent the phase relations in the metamaterial calculated from $7.2 \mathrm{GHz}$ to $8.0 \mathrm{GHz}$ with $0.2 \mathrm{GHz}$ step, while the circle with larger radius is the phase relation in air for reference. Apparently, the $n_{\text {eff }}$ of a wave in the metamaterial is much smaller than that in air. No real root (frequency) is apparent below $7 \mathrm{GHz}$ due to the cutoff phenomenon (plasma-like property) of this structure.

The phase relation above provides physical insights into wave propagation at the interface. For easy interpretation, we regard the metamaterial as a homogeneous medium with a refractive index $n_{e f f}$. The incident and transmitted angles in the medium and air are denoted by $\theta_{i}$ and $\theta_{t}$ respectively. According to Snell's law, the relation between the incident and transmitted angles is as follows.

$$
n_{e f f} \sin \theta_{i}=\sin \theta_{t}
$$

Since $n_{\text {eff }} \ll 1$, the transmitted angle $\left(\theta_{t}\right)$ must be near zero. This means that the transmitting wave is likely perpendicular to the interface between the two media.

\section{B. Effective Medium Approach for Extracting the Uniform Transmission Line Parameters}

Nicolson and Ross proposed a method for determining the permittivity and permeability of a homogeneous material using time-domain measurements in 1970 [16]. The scattering property of a homogeneous material can be determined by calculating its $z$ and $n$ from reflectionand transmission-coefficients. Here $z$ refers to the wave impedance normalized to $377 \Omega$ inside metamaterial whereas $n$ refers to the effective refractive index. The effective medium approach extends same idea of characterization to metamaterial when it is regarded as a homogeneous material. For better characterization, many researchers have attempted to improve the retrieval processes. Existing methods [17]-[20] use the scattering parameters of a metamaterial slab under the normal incidence of a plane wave. This study performs the parameter retrieval analysis on a $3 \mathrm{D}$ fishnet structure with a periodic arrangement. However, the retrieval process may fail for several reasons such as inaccurate estimates of the effective thickness, relatively small scattering parameters, or incorrect determination of the branch index of the refractive index $n$. To avoid these issues, this study chooses a robust method [11] for effective parameter extraction. The effective permittivity and permeability are calculated from $\mu=n z$ and $\epsilon=n / z$.

Since metamaterial is a passive medium, the signs of computed $z$ and $n$ must satisfy the requirement

$$
\begin{aligned}
\operatorname{Re}\{z\} & \geq 0 \\
\operatorname{Im}\{n\} & \geq 0 .
\end{aligned}
$$

Thus $z$ and $n$ can be determined independently by

$$
\begin{aligned}
z= & \pm \sqrt{\frac{\left(1+S_{11}\right)^{2}-S_{21}^{2}}{\left(1-S_{11}\right)^{2}-S_{21}^{2}}} \\
e^{-j k_{o} n d}= & X \pm j \sqrt{1-X^{2}} \\
n= & \frac{1}{k_{o} d}\left\{-\left[\operatorname{Im}\left\{\ln \left(e^{-j k_{o} n d}\right)\right\}+2 m \pi\right]\right. \\
& \left.+j\left[\operatorname{Re}\left\{\ln \left(e^{-j k_{o} n d}\right)\right\}\right]\right\}
\end{aligned}
$$

where $X=\left(1-S_{11}^{2}+S_{21}^{2}\right) / 2 S_{21}, S_{11}$ and $S_{21}$ individually represent the reflection- and transmission-coefficients. The terms $k_{o}$ and $d$ are the free-space wave-number and slab thickness, respectively. Since this study extracts effective parameters of the fishnet-type metamaterial under the normal incidence of a TEM wave in a parallel-plate waveguide of height $h$, the analysis can be simplified by considering only the unit cell inside a waveguide with two horizontal PEC walls and two vertical PMC walls. Solving (6) directly may lead to an ambiguous result because the logarithmic function itself is multi-valued. Therefore, the choice of branch index $m$ is critical and affects the real part of $n$. Nevertheless, the continuity of both permittivity and permeability as functions of frequency is an essential condition that helps determine the branch index. This study employs an iterative method to address this problem, as noted in [11, sect. D].

The constitutive effective parameters of metamaterial are shown in Fig. 3 using (4)-(6). The plasma-like dielectric function is negative for the operational frequency below $7 \mathrm{GHz}$, while the effective permeability is positive. Consequently, the wave should have a pure imaginary number of propagation constant (refractive index) as depicted in (c), exhibiting the below-cutoff phenomenon. Interestingly, the effective refractive index is zero at $7 \mathrm{GHz}$, while the impedance is extremely high. Once the wave is excited in the effective medium at 7 $\mathrm{GHz}$, the high wave impedance causes a strong reflection from the interface between the effective medium and air. Specifically, the wave impedance inside metamaterial is difficult to match the free-pace wave impedance, and therefore we append the flared opening between fishnet structure and free space as an impedance transformer. The effective medium approach can properly model the complicated structure as a 


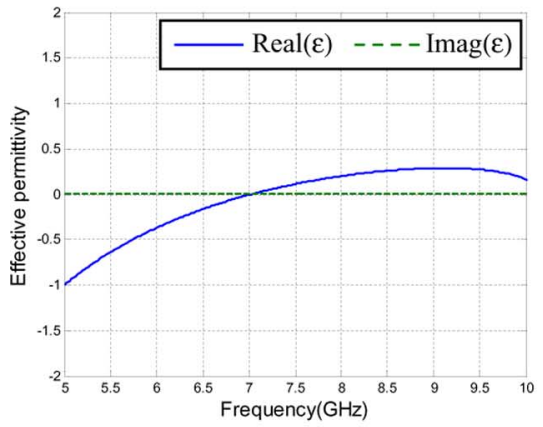

(a)

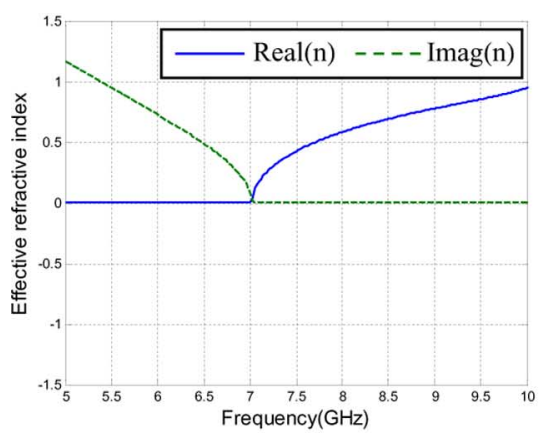

(c)

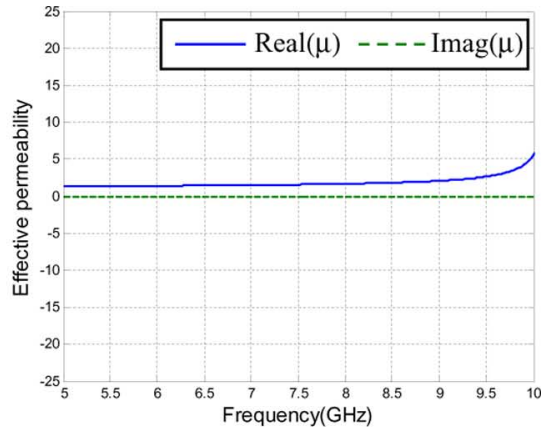

(b)

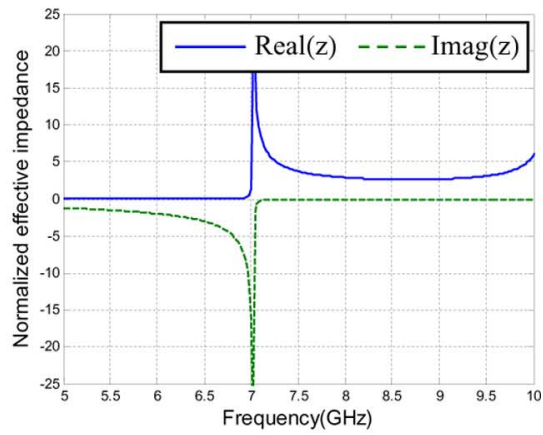

(d)

Fig. 3. Retrieved constitutive effective parameters of the $3 \mathrm{D}$ fishnet metamaterial: (a) permittivity, (b) permeability, (c) refractive index, (d) normalized impedance.

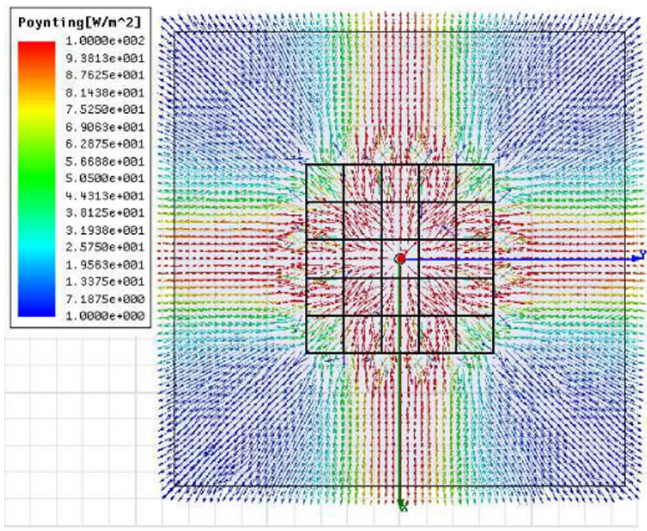

(a)

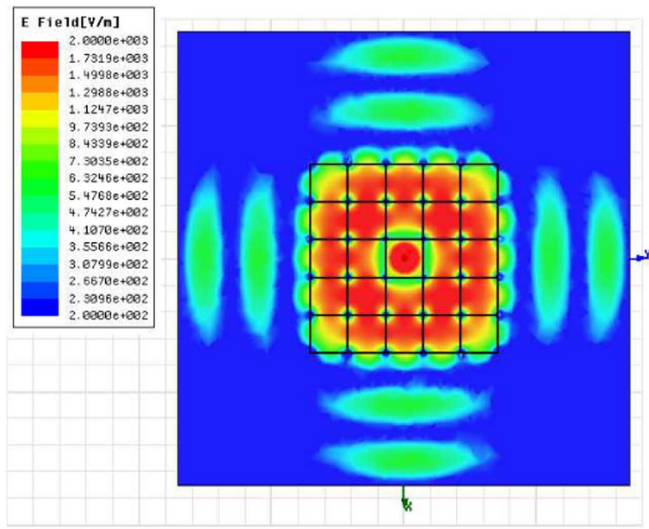

(b)

Fig. 4. (a) Poynting vector distribution in the structure with metamaterial and (b) Electric-field strength in the structure with metamaterial.

uniform dielectric medium, providing uniform transmission-line parameters when the operational wavelength exceeds the period of a periodic structure.

After identifying the phase- and dispersion-relation of eigen-waves supported in the metamaterial, it is possible to observe the physical picture of wave process. By properly tuning the length of the monopole (line source) in the metamaterial, it is possible to diminish the reflection between the coaxial probe and whole structure. The refractive wave should then be normal to the metamaterial surface based on previous predictions. Therefore, this study calculates the electric-field distribution and vectorized Poynting power in the parallel-plate region to verify this conjecture. Fig. 4(a) shows that the refracted wave leaving the metamaterial (highlighted by a grid region) tends to be perpendicular to the metamaterial surface, particularly around the central part. On the other hand, the wave propagates with a small angle deviated from direction perpendicular to side faces of the fishnet structure in the horizontal plane for the reason that the effective refractive index of metamaterial is not perfect but approximate zero in our design.

\section{Radiation Characteristics}

This study demonstrates the spatial power-splitting characteristics of the original structure equipped with a flared opening on each of the four output ports. The flared opening in this design enhances the directivity of the original structure. As is well known in microwave and millimeter wave engineering, an $E$-plane horn antenna uses a flared opening to taper its dominant waveguide mode from the waveguide end to a large opening while maintaining field uniformity. This study uses the same design to obtain a uniform electric-field distribution on the flared opening. The radiation patterns along the $X-Y$ and $X-Z$ plane were measured in an anechoic chamber using a vector network analyzer (HP 8722 D) and standard horn antennas. 


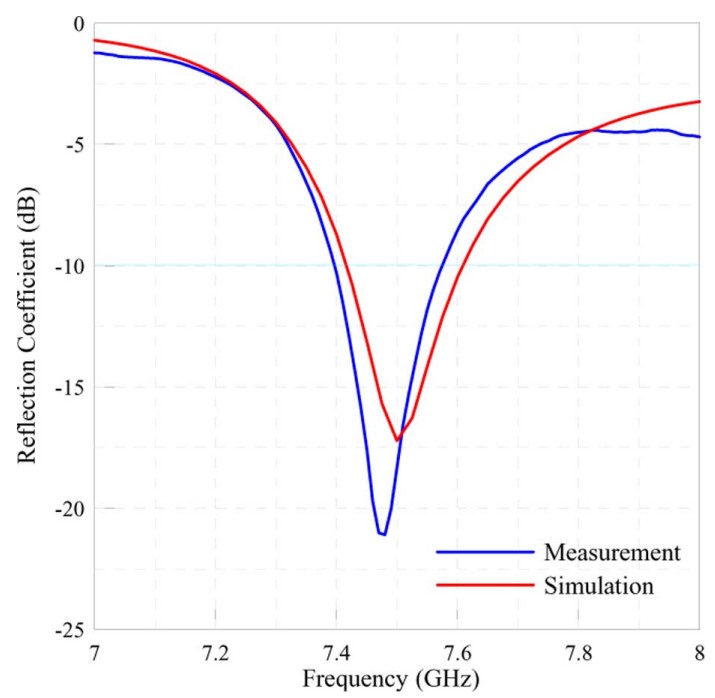

Fig. 5. Reflection coefficient (in $\mathrm{dB}$ ) versus frequency of this spatial beamsplitting structure.

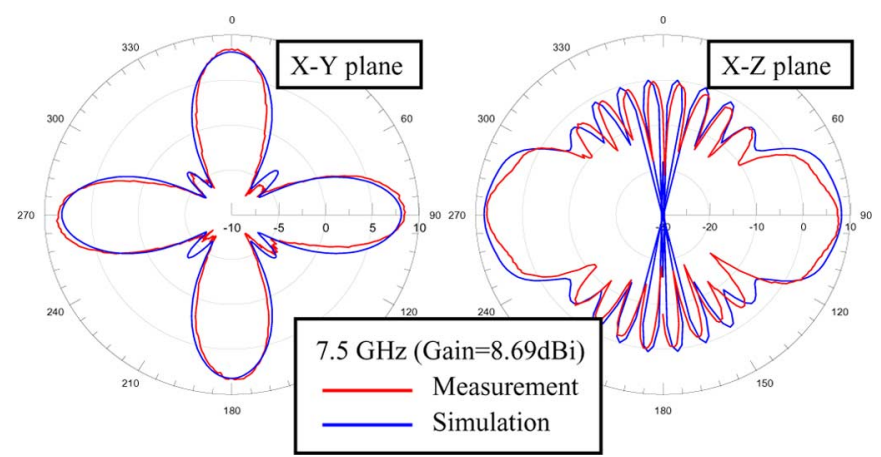

Fig. 6. Radiation patterns of the spatial beam-splitting structure on $X-Z$ and $X-Z$ planes at $7.5 \mathrm{GHz}$.

This study includes the numerical simulations to determine far-field radiation patterns and verify the proposed design concept. Fig. 5 shows the reflection coefficient having bandwidth of $0.2 \mathrm{GHz}$ with VSWR less than 2. Fig. 6 shows both measured and simulated co-polarization patterns. Due to the smaller dimension along the flared-opening direction compared to the width along the $x$ - (or $y$-) axis, the beam pattern has a wider beam-width along the $X-Z$ plane. Although not shown in the figures, measurements of the cross-polarization patterns are much smaller than those of co-polarization. These figures show that the four cone-type beam patterns indeed can serve as a spatial beam splitter to distribute the input power in four directions. The excellent agreement between numerical and measured results confirm the near-zero-refractive-index properties of the metamaterial.

\section{CONCLUSION}

This communication develops an artificial medium based on the 3 D fishnet structure. Using the effective medium approach, the proposed approach regards a $2 \mathrm{D}$ periodic structure as a homogeneous medium and extracts its effective parameters, including the permittivity, permeability, refractive index, and wave impedance. Interestingly, the medium possesses a near-zero refractive index. A spatial beam splitter incorporating this metamaterial was developed to distribute the input power in four directions. To determine the underlying physics of wave process in the metamaterial, this study analyzes the dispersion characteristics of the fishnet structure. In addition, the Poynting vector and electric-field distribution in the metamaterial verifies the property of near-zero refractive index. The excellent agreement between measured and calculated results confirms the design concept of this study.

\section{REFERENCES}

[1] N. Engheta and R. W. Ziolkowski, "Introduction, history and selected topics in fundamental theories of metamaterials," in Metamaterials: Physics and Engineering Explorations, N. Engheta and R. W. Ziolkowski, Eds. Piscataway/Hoboken, NJ: IEEE Press/Wiley, 2006, ch. 1, pp. 5-41.

[2] N. Engheta, A. Alú, R. W. Ziolkowski, and A. Erentok, "Fundamentals of waveguide and antenna applications involving DNG and SNG metamaterials," in Metamaterials: Physics and Engineering Explorations, N. Engheta and R. W. Ziolkowski, Eds. Piscataway/Hoboken, NJ: IEEE Press/Wiley, 2006, ch. 2, pp. 43-85.

[3] R. W. Ziolkowski, "Propagation in and scattering from a matched metamaterial having a zero index of refraction," Phys. Rev. E, vol. 70, no. 4, pp. 046608-046608, 2004.

[4] J. B. Pendry, A. J. Holden, D. J. Robbins, and W. J. Stewart, "Low frequency plasmons in thin-wire structures," J. Phys. Condens. Matter, vol. 10, no. 22, pp. 4785-4809, 1998.

[5] N. Garcia, E. V. Ponizovskaya, and J. Q. Xiao, "Zero permittivity materials: Band gaps at the visible," Appl. Phys. Lett., vol. 80, no. 7, pp. 1120-1122, 2002.

[6] J. B. Pendry, A. J. Holden, D. J. Robbins, and W. J. Stewart, "Magnetism from conductors and enhanced nonlinear phenomena," IEEE Trans. Microw. Theory Tech., vol. 47, no. 11, pp. 2075-2084, 1999.

[7] R. A. Shelby, D. R. Smith, and S. Schultz, "Experimental verification of a negative index of refraction," Science, vol. 292, no. 5514, pp. 77-79, 2001.

[8] S. Zhang, W. Fan, K. J. Malloy, S. R. Brueck, N. C. Panoiu, and R. M. Osgood, "Near-infrared double negative metamaterials," Opt. Express, vol. 13, no. 13, pp. 4922-4930, 2005.

[9] S. Zhang, W. Fan, N. C. Panoiu, K. J. Malloy, R. M. Osgood, and S. R. Brueck, "Optical negative-index bulk metamaterials consisting of $2 \mathrm{~d}$ perforated metal-dielectric stacks," Opt. Express, vol. 14, pp. 6778-6787, 2006.

[10] J. Valentine, S. Zhang, T. Zentgraf, E. Ulin-Avila, D. Genov, G. Bartal, and X. Zhang, "Three-dimensional optical metamaterial with a negative refractive index," Nature, vol. 455, no. 7211, pp. 376-379, 2008.

[11] X. Chen, T. M. Grzegorczyk, B.-I. Wu, J. Pacheco, and J. A. Kong, "Robust method to retrieve the constitutive effective parameters of metamaterials," Phys. Rev. E, vol. 70, no. 1, pp. 016608-016608, 2004.

[12] S. Enoch, G. Tayeb, P. Sabouroux, N. Guérin, and P. Vincent, "A metamaterial for directive emission," Phys. Rev. Lett., vol. 89, no. 21, pp. 213902-213902, 2002.

[13] A. Alù, M. Silveirinha, A. Salandrino, and N. Engheta, "Epsilon-nearzero metamaterials and electromagnetic sources: Tailoring the radiation phase pattern," Phys. Rev. B, vol. 75, no. 15, pp. 155410-155410, 2007.

[14] M. Notomi, "Theory of light propagation in strongly modulated photonic crystals: Refractionlike behavior in the vicinity of the photonic band gap," Phys. Rev. B, vol. 62, no. 16, pp. 10696-10705, 2000.

[15] B.-I. Wu, W. Wang, J. Pacheco, X. Chen, T. M. Grzegorczyk, and J. A. Kong, "A study of using metamaterials as antenna substrate to enhance gain," PIER, vol. 51, pp. 295-328, 2005.

[16] A. M. Nicolson and G. F. Ross, "Measurement of the intrinsic properties of materials by time-domain techniques," IEEE Trans. Instrum. Meas., vol. 19, no. 4, pp. 377-382, 1970.

[17] J. Baker-Jarvis, E. Vanzura, and W. Kissick, "Improved technique for determining complex permittivity with the transmission/reflection method," IEEE Trans. Microw. Theory Tech., vol. 38, no. 8, pp. 1096-1103, Aug. 1990.

[18] D. R. Smith, S. Schultz, P. Markoš, and C. M. Soukoulis, "Determination of effective permittivity and permeability of metamaterials from reflection and transmission coefficients," Phys. Rev. B, vol. 65, no. 19, pp. 195104-195104, 2002.

[19] P. Markoš and C. Soukoulis, "Transmission properties and effective electromagnetic parameters of double negative metamaterials," Opt. Express, vol. 11, no. 7, pp. 649-661, 2003.

[20] R. W. Ziolkowski, "Design, fabrication, and testing of double negative metamaterials," IEEE Trans. Antennas Propag., vol. 51, no. 7, pp. 1516-1529, Jul. 2003. 